\title{
Response to "Contemporary Outcomes of Patients with Isolated Bilateral Renal Agenesis with and without Fetal Intervention" by RAFT Investigators
}

\author{
Eric Bradley Jelin ${ }^{\mathrm{a}}$ Meredith Atkinson ${ }^{\mathrm{b}}$ Amaris Keiser ${ }^{\mathrm{c}}$ Yair J. Blumenfeld ${ }^{\mathrm{d}}$ \\ Ahmet Alexander Baschat ${ }^{\mathrm{e}}$

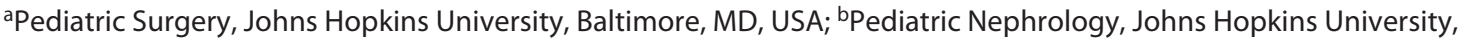 \\ Baltimore, MD, USA; ${ }^{C}$ Neonatology, Johns Hopkins University, Baltimore, MD, USA; ${ }^{d}$ Maternal Fetal Medicine, \\ Stanford University, Palo Alto, CA, USA; ${ }^{\text {C} C e n t e r ~ f o r ~ F e t a l ~ T h e r a p y, ~ J o h n s ~ H o p k i n s ~ U n i v e r s i t y, ~ B a l t i m o r e, ~ M D, ~ U S A ~}$
}

\section{Dear Editor,}

We read with great interest the recently published article entitled "Contemporary Outcomes of Patients with Isolated Bilateral Renal Agenesis with and without Fetal Intervention" by Riddle and colleagues [1]. In this singlecenter experience of fetuses diagnosed with bilateral renal agenesis (BRA) over a 13-year period, the investigators document many granular details about maternal and fetal demographic characteristics and the prenatal, perinatal, and neonatal clinical course, which greatly enriches the current fledgling literature. Furthermore, the inclusion of patients receiving both amnioinfusions as well as those opting for expectant management without in utero intervention provides an opportunity to better understand the postnatal clinical trajectories for both groups. We agree with the authors" conclusion that "any further treatment of this population should only be performed with careful institutional review board and ethical oversight," a conclusion which reaffirms the previously published consensus statement of a national multidisciplinary work group that in 2018 evaluated complex ethical concerns associated with the use of serial amnioinfusions for BRA [2]. However, we respectfully challenge the authors' followup conclusion that future research "should focus more on

karger@karger.com

www.karger.com/fdt

(c) 2020 S. Karger AG, Basel

Karger! standardizing the approach to the neonatal care of these infants." The presented study lacks standardization of prenatal management and, therefore, has limited ability to produce generalizable knowledge on postnatal management issues.

Although there was undoubtedly variability in the postnatal management of the 14 live-born infants - including baseline practice changes over the 15-year period of data collection and the use of multiple delivery hospitals - we note the equally problematic variability in the prenatal management of the intervention patients. Specifically, of the 8 patients with fetal BRA who received serial infusions, 3 did not have a formal genetic evaluation and 2 had an unknown gestational age at last amnioinfusion. Furthermore, at maximum 5 of the 8, and perhaps fewer of the intervention group, delivered at the treating fetal center. These methodologic issues coupled with the small sample size make drawing conclusion about fetal therapy technique, complication rate, maternal safety, and neonatal survivorship untenable.

The NICHD-funded prospective Renal Anhydramnios Fetal Therapy (RAFT) trial (NCT03101891) [3,4] is the "North American Fetal Therapy Network Collaborative ... prospective study enrolling patients whose fetuses

Eric Bradley Jelin

Department of Surgery, Division of Pediatric Surgery, Johns Hopkins University 1800 Orleans Street

Baltimore, MD 21212 (USA)

ejelin1@jhmi.edu 
have BRA" to which we believe the authors refer. This trial is currently enrolling patients and is slated to include 35 fetuses with prenatally diagnosed BRA, with a primary outcome of neonatal respiratory survival to successful dialysis. A standardized set of eligibility criteria and standardized technical guidelines for the percutaneous amnioinfusion procedure have been developed and a data safety monitoring board convened as part of this multisite prospective trial. This study is specifically powered to determine the pulmonary survival rate for neonates with isolated BRA (supported by genetic evaluation) who receive percutaneous serial amnioinfusions. This question must be prospectively answered before evaluation and ultimately standardization of optimal postnatal care can occur. The cart cannot be put before the proverbial horse. The RAFT trial is carefully monitoring the postnatal management of these neonates, who will be delivered at participating RAFT centers, all of which have the ability to provide comprehensive neonatal intensive care and utilize the most current neonatal dialysis techniques. Hy- potheses about how to standardize these techniques will be relevant once pulmonary survivorship is demonstrated among a significant number of uniformly selected patients who have been treated with a standard approach to prenatal amnioinfusion.

\section{Conflict of Interest Statement}

The authors have no conflicts of interest to declare.

\section{Funding Sources}

The authors are funded by the National Institute of Health R01HD100540.

\section{Author Contributions}

All authors contributed to the conception, drafting, and critical revision of this letter.

\section{References}

1 Riddle S, Habli M, Tabbah S, Lim FY, Minges $\mathrm{M}$, Kingma P, et al. Contemporary outcomes of patients with isolated bilateral renal agenesis with and without fetal intervention. Fetal Diagn Ther. 2020 Jun:1-7.

2 Sugarman J, Anderson J, Baschat AA, Herrera Beutler J, Bienstock JL, Bunchman TE, et al.
Ethical considerations concerning amnioinfusions for treating fetal bilateral renal agenesis. Obstet Gynecol. 2018;131(1):130-4.

3 O'Hare EM, Jelin AC, Miller JL, Ruano R, Atkinson MA, Baschat AA, et al. Amnioinfusions to treat early onset anhydramnios caused by renal anomalies: background and rationale for the renal anhydramnios fetal therapy trial. Fetal Diagn Ther. 2019;45(6):365-72.

4 Jelin AC, Sagaser KG, Forster KR, Ibekwe T, Norton ME, Jelin EB. Etiology and management of early pregnancy renal anhydramnios: is there a place for serial amnioinfusions? Prenat Diagn. 2020 Feb;40(5):528-37. 